\title{
Convexity and Spirallikeness Conditions for Two New General Integral Operators
}

\author{
H. Özlem Güney ${ }^{1}$ and Serap Bulut ${ }^{2}$ \\ ${ }^{1}$ Department of Mathematics, Faculty of Science, Dicle University, 21280 Diyarbakir, Turkey \\ ${ }^{2}$ Civil Aviation College, Kocaeli University, Arslanbey Campus, 41285 Kocaeli, Turkey
}

Correspondence should be addressed to Serap Bulut; bulutserap@yahoo.com

Received 3 January 2013; Accepted 11 April 2013

Academic Editor: Majid Soleimani-damaneh

Copyright (c) 2013 H. Ö. Güney and S. Bulut. This is an open access article distributed under the Creative Commons Attribution License, which permits unrestricted use, distribution, and reproduction in any medium, provided the original work is properly cited.

We define two new general integral operators for certain analytic functions in the unit disc $\mathbb{U}$ and give some sufficient conditions for these integral operators on some subclasses of analytic functions.

\section{Introduction}

Let $\mathscr{A}_{p}(n)$ denote the class of all functions of the form

$$
f(z)=z^{p}+\sum_{k=p+n}^{\infty} a_{k} z^{k} \quad(p, n \in \mathbb{N}=\{1,2, \ldots\})
$$

which is analytic in the open unit disc $\mathbb{U}=\{z \in \mathbb{C}:|z|<1\}$.

In particular, we set

$$
\mathscr{A}_{p}(1):=\mathscr{A}_{p}, \quad \mathscr{A}_{1}(1)=\mathscr{A}_{1}:=\mathscr{A} .
$$

The fractional derivative of order $\alpha$ is defined, for a function $f$, by

$$
D_{z}^{\alpha} f(z)=\frac{1}{\Gamma(1-\alpha)} \frac{d}{d z} \int_{0}^{z} \frac{f(\zeta)}{(z-\zeta)^{\alpha}} d \zeta \quad(0 \leq \alpha<1)
$$

where the function $f$ is analytic in a simply connected region of the complex $z$-plane containing the origin, and the multiplicity of $(z-\zeta)^{-\alpha}$ is removed by requiring $\log (z-\zeta)$ to be real when $z-\zeta>0$.

It readily follows from (3) that

$$
D_{z}^{\alpha} z^{k}=\frac{\Gamma(k+1)}{\Gamma(k-\alpha+1)} z^{k-\alpha} \quad(0 \leq \alpha<1, k \in \mathbb{N}) .
$$

Using the operator $\Omega_{p}^{\alpha}: \mathscr{A}_{p}(n) \rightarrow \mathscr{A}_{p}(n)$,

$$
\begin{aligned}
& \Omega_{p}^{\alpha} f(z)= \frac{\Gamma(p-\alpha+1)}{\Gamma(p+1)} z^{\alpha} D_{z}^{\alpha} f(z), \\
&=z^{p}+\sum_{k=p+n}^{\infty} \frac{\Gamma(k+1) \Gamma(p-\alpha+1)}{\Gamma(p+1) \Gamma(k-\alpha+1)} a_{k} z^{k}, \\
& \alpha \neq p+1, p+2, p+3, \ldots
\end{aligned}
$$

Bulut [1] defined the general differential operator $D_{\lambda, l, p}^{m, \alpha}$, namely, the generalization of the generalized Al-Oboudi differential operator as follows:

$$
\begin{gathered}
D^{0} f(z)=f(z), \\
D_{\lambda, l, p}^{1, \alpha} f(z)=\frac{p-\lambda p+l}{p+l} \Omega_{p}^{\alpha} f(z)+\frac{\lambda}{p+l} z\left(\Omega_{p}^{\alpha} f(z)\right)^{\prime} \\
=D_{\lambda, l, p}^{\alpha} f(z) \quad(\lambda, l \geq 0,0 \leq \alpha<1), \\
D_{\lambda, l, p}^{2, \alpha} f(z)=D_{\lambda, l, p}^{\alpha}\left(D_{\lambda, l, p}^{1, \alpha} f(z)\right),
\end{gathered}
$$


If $f$ is given by (1), then by (5) and (6), we see that

$$
\begin{array}{r}
D_{\lambda, l, p}^{m, \alpha} f(z)=z^{p}+\sum_{k=p+n}^{\infty} \Psi_{k, m}(\alpha, \lambda, l, p) a_{k} z^{k} \\
\left(m \in \mathbb{N}_{0}=\mathbb{N} \cup\{0\}\right),
\end{array}
$$

where

$$
\begin{aligned}
\Psi_{k, m}(\alpha, \lambda, l, p) & \\
& =\left[\frac{\Gamma(k+1) \Gamma(p-\alpha+1)}{\Gamma(p+1) \Gamma(k-\alpha+1)} \frac{p+\lambda(k-p)+l}{p+l}\right]^{m} .
\end{aligned}
$$

By using the operator $D_{\lambda, l, p}^{m, \alpha}$ defined by (7), we introduce the new classes $\mathcal{U} \mathcal{S}_{\alpha, \lambda, l}^{m, p, n}(\delta, \beta, b)$ and $\mathcal{U}_{\alpha, \lambda, l}^{m, p, n}(\delta, \beta, b)$ as follows.

Definition 1. A function $f \in \mathscr{A}_{p}(n)$ is in the class $\mathcal{U} \mathcal{S}_{\alpha, \lambda, l}^{m, p, n}(\delta, \beta, b)$ if and only if $f$ satisfies

$$
\begin{aligned}
\mathfrak{R}\left\{p+\frac{1}{b}\left(\frac{z\left(D_{\lambda, l, p}^{m, \alpha} f(z)\right)^{\prime}}{D_{\lambda, l, p}^{m, \alpha} f(z)}-p\right)\right\} \\
>\delta\left|\frac{1}{b}\left(\frac{z\left(D_{\lambda, l, p}^{m, \alpha} f(z)\right)^{\prime}}{D_{\lambda, l, p}^{m, \alpha} f(z)}-p\right)\right|+\beta,
\end{aligned}
$$

where $z \in \mathbb{U}, b \in \mathbb{C}-\{0\}, \delta \geq 0,0 \leq \beta<p$ and $D_{\lambda, l, p}^{m, \alpha}$ is defined by (7).

Definition 2. A function $f \in \mathscr{A}_{p}(n)$ is in the class $\mathcal{U} \mathscr{K}_{\alpha, \lambda, l}^{m, p, n}(\delta, \beta, b)$ if and only if $f$ satisfies

$$
\begin{gathered}
\mathfrak{R}\left\{p+\frac{1}{b}\left(1+\frac{z\left(D_{\lambda, l, p}^{m, \alpha} f(z)\right)^{\prime \prime}}{\left(D_{\lambda, l, p}^{m, \alpha} f(z)\right)^{\prime}}-p\right)\right\} \\
>\delta\left|\frac{1}{b}\left(1+\frac{z\left(D_{\lambda, l, p}^{m, \alpha} f(z)\right)^{\prime \prime}}{\left(D_{\lambda, l, p}^{m, \alpha} f(z)\right)^{\prime}}-p\right)\right|+\beta,
\end{gathered}
$$

where $z \in \mathbb{U}, b \in \mathbb{C}-\{0\}, \delta \geq 0,0 \leq \beta<p$, and $D_{\lambda, l, p}^{m, \alpha}$ is defined by (7).

Note that

$$
f \in \mathcal{U} \mathscr{K}_{\alpha, \lambda, l}^{m, p, n}(\delta, \beta, b) \Longleftrightarrow \frac{1}{p} z f^{\prime} \in \mathcal{U} \mathcal{S}_{\alpha, \lambda, l}^{m, p, n}(\delta, \beta, b) .
$$

Remark 3. (i) For $\delta=0$, we have the classes

$$
\begin{aligned}
& \mathcal{U} \mathcal{S}_{\alpha, \lambda, l}^{m, p, n}(0, \beta, b) \equiv \mathcal{S}_{\alpha, \lambda, l}^{m, p, n}(\beta, b), \\
& \mathcal{U} \mathscr{K}_{\alpha, \lambda, l}^{m, p, n}(0, \beta, b) \equiv \mathscr{K}_{\alpha, \lambda, l}^{m, p, n}(\beta, b)
\end{aligned}
$$

introduced and studied by Bulut [1]. In particular, we get the classes

$$
\begin{aligned}
& \mathcal{U} \mathcal{S}_{\alpha, \lambda, 0}^{m, 1,1}(0, \beta, b) \equiv \mathcal{S}_{\alpha, \lambda}^{m}(\beta, b), \\
& \mathcal{U} \mathscr{K}_{\alpha, \lambda, 0}^{m, 1,1}(0, \beta, b) \equiv \mathscr{K}_{\alpha, \lambda}^{m}(\beta, b)
\end{aligned}
$$

introduced and studied by Bulut [2].

(ii) For $\delta=0$ and $\beta=0$, we have the classes

$$
\begin{gathered}
\mathcal{U} \mathcal{S}_{\alpha, \lambda, l}^{m, p, n}(0,0, b) \equiv \mathcal{S}_{\alpha, \lambda, l}^{m, p, n}(b), \\
\mathcal{U} \mathscr{K}_{\alpha, \lambda, l}^{m, p, n}(0,0, b) \equiv \mathscr{K}_{\alpha, \lambda, l}^{m, p, n}(b) .
\end{gathered}
$$

In particular, we set

$$
\begin{aligned}
& \mathcal{U} \mathcal{S}_{\alpha, \lambda, l}^{0, p, n}(0,0, b) \equiv \mathcal{S}^{p, n}(b), \\
& \mathcal{U} \mathscr{K}_{\alpha, \lambda, l}^{0, p, n}(0,0, b) \equiv \mathscr{K}^{p, n}(b)
\end{aligned}
$$

for the $p$-valently starlike and $p$-valently convex functions with complex order $b(b \in \mathbb{C}-\{0\})$, respectively.

(iii) For $m=0$, we have the classes

$$
\begin{aligned}
\mathscr{U}_{\alpha, \lambda, l}^{0, p, n}(\delta, \beta, b) & \\
& \equiv \mathcal{S}^{p, n}(\delta, \beta, b) \\
& =\left\{f \in \mathscr{A}_{p}(n): \mathfrak{R}\left\{p+\frac{1}{b}\left(\frac{z f^{\prime}(z)}{f(z)}-p\right)\right\}\right. \\
& \left.>\delta\left|\frac{1}{b}\left(\frac{z f^{\prime}(z)}{f(z)}-p\right)\right|+\beta\right\}, \\
& \equiv \mathscr{K}_{\alpha, \lambda, l}^{0, p, n}(\delta, \beta, b) \\
& =\left\{f \in \mathscr{K}_{p}^{p, n}(\delta, \beta, b): \mathfrak{R}\left\{p+\frac{1}{b}\left(1+\frac{z f^{\prime \prime}(z)}{f^{\prime}(z)}-p\right)\right\}\right. \\
> & \left.\delta\left|\frac{1}{b}\left(1+\frac{z f^{\prime \prime}(z)}{f^{\prime}(z)}-p\right)\right|+\beta\right\} .
\end{aligned}
$$

In particular, we set

$$
\begin{aligned}
\mathcal{U} \mathcal{S}_{\alpha, \lambda, l}^{0,1,1}(\delta, \beta, b) & \equiv \mathcal{S}(\delta, \beta, b), \\
\mathcal{U} \mathscr{K}_{\alpha, \lambda, l}^{0,1,1}(\delta, \beta, b) & \equiv \mathscr{K}(\delta, \beta, b) .
\end{aligned}
$$

Also, we have the classes

$$
\begin{aligned}
& \mathcal{U} \mathcal{S}_{\alpha, \lambda, l}^{0,1,1}(\delta, \beta, 1) \equiv \mathcal{S} \mathscr{D}(\delta, \beta), \\
& \mathcal{U} \mathscr{K}_{\alpha, \lambda, l}^{0,1,1}(\delta, \beta, 1) \equiv \mathscr{K} \mathscr{D}(\delta, \beta)
\end{aligned}
$$

introduced and studied by Shams et al. [3]. 
(iv) For $\delta=0$ and $m=0$, we have the classes

$$
\begin{aligned}
\mathscr{U} & \mathcal{S}_{\alpha, \lambda, l}^{0, p, n}(0, \beta, b) \\
& \equiv \mathcal{S}^{p, n}(\beta, b) \\
& =\left\{f \in \mathscr{A}_{p}(n): \Re\left\{p+\frac{1}{b}\left(\frac{z f^{\prime}(z)}{f(z)}-p\right)\right\}>\beta\right\}, \\
\mathscr{U}_{\alpha, \lambda, l}^{0, p, n}(0, \beta, b) & \equiv \mathscr{K}^{p, n}(\beta, b) \\
& =\left\{f \in \mathscr{A}_{p}(n): \Re\left\{p+\frac{1}{b}\left(1+\frac{z f^{\prime \prime}(z)}{f^{\prime}(z)}-p\right)\right\}>\beta\right\}
\end{aligned}
$$

of $p$-valently starlike and $p$-valently convex functions with complex order $b(b \in \mathbb{C}-\{0\})$ and type $\beta(0 \leq \beta<p)$, respectively. In particular, we set

$$
\begin{gathered}
\mathscr{U} \mathcal{S}_{\alpha, \lambda, l}^{0,1,1}(0, \beta, b) \equiv \mathcal{S}(\beta, b), \\
\mathcal{U} \mathscr{K}_{\alpha, \lambda, l}^{0,1,1}(0, \beta, b) \equiv \mathscr{K}(\beta, b) \quad(b \in \mathbb{C}-\{0\}, 0 \leq \beta<1) .
\end{gathered}
$$

Note that for $b=1$, we have

$$
\mathcal{U} \mathcal{S}_{\alpha, \lambda, l}^{0,1,1}(0, \beta, 1) \equiv \mathcal{S}^{*}(\beta), \quad \mathcal{U} \mathscr{K}_{\alpha, \lambda, l}^{0,1,1}(0, \beta, 1) \equiv \mathscr{K}(\beta)
$$

which are the classes of starlike and convex functions type $\beta(0 \leq \beta<1)$, respectively.

(v) For $\delta=1, m=0, p=n=1$, and $b=1$, we have the classes

$$
\begin{aligned}
& \mathcal{U} \mathcal{S}_{\alpha, \lambda, l}^{0,1,1}(1, \beta, 1) \equiv \mathscr{U} \mathcal{S} \mathscr{T}(\beta), \\
& \mathcal{U} \mathscr{K}_{\alpha, \lambda, l}^{0,1,1}(\delta, \beta, 1) \equiv \mathcal{U} \mathscr{C} \mathscr{V}(\beta)
\end{aligned}
$$

of uniformly starlike and uniformly convex functions of order $\beta(0 \leq \beta<1)$, respectively, investigated by Rønning [4]. In particular, we get the classes

$$
\mathscr{U} \mathcal{S}_{\alpha, \lambda, l}^{0,1,1}(1,0,1) \equiv \mathcal{U} \mathcal{S} \mathscr{T}, \quad \mathscr{U} \mathscr{K}_{\alpha, \lambda, l}^{0,1,1}(\delta, \beta, 1) \equiv \mathscr{U} \mathscr{C} \mathscr{V}
$$

of uniformly starlike and uniformly convex functions, respectively, first defined by Goodman [5].
Remark 4. If we set $\delta=0$ and $b=e^{-i \theta} \cos \theta(|\theta|<\pi / 2)$ in Definitions 1 and 2, then we have the classes

$$
\begin{aligned}
& \mathcal{S}_{\alpha, \lambda, l}^{m, p, n}(\beta, \theta) \\
& =\left\{f \in \mathscr{A}_{p}(n): \mathfrak{R}\left\{e^{i \theta} \frac{z\left(D_{\lambda, l, p}^{m, \alpha} f(z)\right)^{\prime}}{D_{\lambda, l, p}^{m, \alpha} f(z)}\right\}\right. \\
& >\beta \cos \theta, 0 \leq \beta<p\}, \\
& \mathscr{K}_{\alpha, \lambda, l}^{m, p, n}(\beta, \theta) \\
& =\left\{f \in \mathscr{A}_{p}(n): \mathfrak{R}\left\{e^{i \theta}\left(1+\frac{z\left(D_{\lambda, l, p}^{m, \alpha} f(z)\right)^{\prime \prime}}{\left(D_{\lambda, l, p}^{m, \alpha} f(z)\right)^{\prime}}\right)\right\}\right. \\
& >\beta \cos \theta, 0 \leq \beta<p\} \text {, }
\end{aligned}
$$

respectively. Also, we put

$$
\mathcal{S}_{\alpha, \lambda, l}^{0, p, n}(\beta, \theta) \equiv \mathcal{S}^{p, n}(\beta, \theta), \quad \mathscr{K}_{\alpha, \lambda, l}^{0, p, n}(\beta, \theta) \equiv \mathscr{K}^{p, n}(\beta, \theta) .
$$

In particular, we set

$$
\begin{aligned}
& \mathcal{S}_{\alpha, \lambda, l}^{0,1,1}(\beta, \theta) \equiv \mathcal{S}_{\beta}^{\theta} \quad\left(|\theta|<\frac{\pi}{2}, 0 \leq \beta<1\right), \\
& \mathscr{K}_{\alpha, \lambda, l}^{0,1,1}(\beta, \theta) \equiv \mathscr{K}_{\beta}^{\theta} \quad\left(|\theta|<\frac{\pi}{2}, 0 \leq \beta<1\right)
\end{aligned}
$$

for the the class of $\theta$-spirallike functions of order $\beta$ [6], and the class of $\theta$-Robertson functions of order $\beta$ [7], respectively. We now define the following general integral operators.

Definition 5. Let $\eta \in \mathbb{N}, m=\left(m_{1}, \ldots, m_{\eta}\right) \in \mathbb{N}_{0}^{\eta}$, and $k=$ $\left(k_{1}, \ldots, k_{\eta}\right) \in \mathbb{R}_{+}^{\eta}$. One defines the following general integral operators:

$$
\begin{gathered}
\mathscr{I}_{p, \eta}^{m, k}: \mathscr{A}_{p}(n)^{\eta} \longrightarrow \mathscr{A}_{p}(n), \\
\mathscr{J}_{p, \eta}^{m, k}\left(f_{1}, \ldots, f_{\eta}\right)=\mathscr{F}_{p, \eta, m, k}, \\
\mathscr{F}_{p, \eta, m, k}(z)=\int_{0}^{z} p t^{p-1} \prod_{j=1}^{\eta}\left(\frac{D_{\lambda, l, p}^{m_{j}, \alpha} f_{j}(t)}{t^{p}}\right)^{k_{j}} d t, \\
\mathscr{J}_{p, \eta}^{m, k}: \mathscr{A}_{p}(n)^{\eta} \longrightarrow \mathscr{A}_{p}(n), \\
\mathscr{J}_{p, \eta}^{m, k}\left(f_{1}, \ldots, f_{\eta}\right)=\mathscr{G}_{p, \eta, m, k}, \\
\mathscr{G}_{p, \eta, m, k}(z)=\int_{0}^{z} p t^{p-1} \prod_{j=1}^{\eta}\left(\frac{\left(D_{\lambda, l, p}^{m_{j}, \alpha} f_{j}(t)\right)^{\prime}}{p t^{p-1}}\right)^{k_{j}} d t,
\end{gathered}
$$


where $z \in \mathbb{U}, f_{j} \in \mathscr{A}_{p}(n)$, and $D_{\lambda, l, p}^{m_{j}, \alpha}$ is defined by (7) for $1 \leq j \leq \eta$.

Remark 6. (i) For $\eta=1, m_{1}=m, k_{1}=k$, and $f_{1}=f$, we have the integral operators

$$
\begin{gathered}
\mathscr{F}_{p, m, k}(z)=\int_{0}^{z} p t^{p-1}\left(\frac{D_{\lambda, l, p}^{m, \alpha} f(t)}{t^{p}}\right)^{k} d t, \\
\mathscr{G}_{p, m, k}(z)=\int_{0}^{z} p t^{p-1}\left(\frac{\left(D_{\lambda, l, p}^{m, \alpha} f(t)\right)^{\prime}}{p^{t^{p-1}}}\right)^{k} d t .
\end{gathered}
$$

(ii) For $\alpha=0, \lambda=1$, and $l=0$, we have the integral operators

$$
\begin{gathered}
\mathscr{F}_{p, n, m, k}(z)=\int_{0}^{z} p t^{p-1}\left(\frac{D^{m_{1}} f_{1}(t)}{t^{p}}\right)^{k_{1}} \cdots\left(\frac{D^{m_{n}} f_{n}(t)}{t^{p}}\right)^{k_{n}} d t, \\
\mathscr{G}_{p, n, m, k}(z) \\
=\int_{0}^{z} p t^{p-1}\left(\frac{\left(D^{m_{1}} f_{1}(t)\right)^{\prime}}{p t^{p-1}}\right)^{k_{1}} \cdots\left(\frac{\left(D^{m_{n}} f_{n}(t)\right)^{\prime}}{p t^{p-1}}\right)^{k_{n}} d t
\end{gathered}
$$

introduced by Saltık et al. [8].

(iii) For $m_{1}=\cdots=m_{\eta}=0$, we have the integral operators

$$
\begin{aligned}
& \mathscr{F}_{p}(z)=\int_{0}^{z} p t^{p-1}\left(\frac{f_{1}(t)}{t^{p}}\right)^{k_{1}} \cdots\left(\frac{f_{n}(t)}{t^{p}}\right)^{k_{n}} d t, \\
& \mathscr{G}_{p}(z)=\int_{0}^{z} p t^{p^{p-1}}\left(\frac{f_{1}^{\prime}(t)}{p t^{p-1}}\right)^{k_{1}} \cdots\left(\frac{f_{n}^{\prime}(t)}{p t^{p^{-1}}}\right)^{k_{n}} d t
\end{aligned}
$$

introduced by Frasin [9].

\section{Sufficient Conditions for}

\section{the Operator $\mathscr{F}_{p, \eta, m, k}$}

Theorem 7. Let $\eta \in \mathbb{N}, m=\left(m_{1}, \ldots, m_{\eta}\right) \in \mathbb{N}_{0}^{\eta}$, and $k=$ $\left(k_{1}, \ldots, k_{\eta}\right) \in \mathbb{R}_{+}^{\eta}$. Also let $b \in \mathbb{C}-\{0\}, \delta_{j} \geq 0,0 \leq \beta_{j}<p$, and $f_{j} \in \mathcal{U} \delta_{\alpha, \lambda, l}^{m_{j}, p, n}\left(\delta_{j}, \beta_{j}, b\right)$ for $1 \leq j \leq \eta$. If

$$
0 \leq p+\sum_{j=1}^{\eta} k_{j}\left(\beta_{j}-p\right)<p,
$$

then the integral operator $\mathscr{F}_{p, \eta, m, k}$, defined by (29), is in the class $\mathscr{K}^{p, n}(\tau, b)$, where

$$
\tau=p+\sum_{j=1}^{\eta} k_{j}\left(\beta_{j}-p\right) .
$$

Proof. From the definition (29), we observe that $\mathscr{F}_{p, \eta, m, k}(z) \in$ $\mathscr{A}_{p}(n)$. On the other hand, it is easy to see that

$$
\left(\mathscr{F}_{p, \eta, m, k}(z)\right)^{\prime}=p z^{p-1} \prod_{j=1}^{\eta}\left(\frac{D_{\lambda, l, p}^{m_{j}, \alpha} f_{j}(z)}{z^{p}}\right)^{k_{j}} .
$$

Differentiating (38) logarithmically and multiplying by $z$, we obtain

$$
\frac{z\left(\mathscr{F}_{p, \eta, m, k}(z)\right)^{\prime \prime}}{\left(\mathscr{F}_{p, \eta, m, k}(z)\right)^{\prime}}=(p-1)+\sum_{j=1}^{\eta} k_{j}\left(\frac{z\left(D_{\lambda, l, p}^{m_{j}, \alpha} f_{j}(t)\right)^{\prime}}{D_{\lambda, l, p}^{m_{j}, \alpha} f_{j}(t)}-p\right)
$$

or equivalently

$$
1+\frac{z\left(\mathscr{F}_{p, \eta, m, k}(z)\right)^{\prime \prime}}{\left(\mathscr{F}_{p, \eta, m, k}(z)\right)^{\prime}}-p=\sum_{j=1}^{\eta} k_{j}\left(\frac{z\left(D_{\lambda, l, p}^{m_{j}, \alpha} f_{j}(t)\right)^{\prime}}{D_{\lambda, l, p}^{m_{j}, \alpha} f_{j}(t)}-p\right) .
$$

Then, by multiplying (40) with $1 / b$, we have

$$
\begin{aligned}
\frac{1}{b}(1 & \left.+\frac{z\left(\mathscr{F}_{p, \eta, m, k}(z)\right)^{\prime \prime}}{\left(\mathscr{F}_{p, \eta, m, k}(z)\right)^{\prime}}-p\right) \\
= & \sum_{j=1}^{\eta} k_{j} \frac{1}{b}\left(\frac{z\left(D_{\lambda, l, p}^{m_{j}, \alpha} f_{j}(t)\right)^{\prime}}{D_{\lambda, l, p}^{m_{j}, \alpha} f_{j}(t)}-p\right)
\end{aligned}
$$

or equivalently

$$
\begin{aligned}
p+\frac{1}{b}\left(1+\frac{z\left(\mathscr{F}_{p, \eta, m, k}(z)\right)^{\prime \prime}}{\left(\mathscr{F}_{p, \eta, m, k}(z)\right)^{\prime}}-p\right) \\
=\sum_{j=1}^{\eta} k_{j}\left(p+\frac{1}{b}\left(\frac{z\left(D_{\lambda, l, p}^{m_{j}, \alpha} f_{j}(t)\right)^{\prime}}{D_{\lambda, l, p}^{m_{j}, \alpha} f_{j}(t)}-p\right)\right) \\
+p-p \sum_{j=1}^{\eta} k_{j} .
\end{aligned}
$$


Since $f_{j} \in \mathcal{U} \mathcal{S}_{\alpha, \lambda, l}^{m_{j}, p, n}\left(\delta_{j}, \beta_{j}, b\right)(1 \leq j \leq \eta)$, we get

$$
\begin{aligned}
\Re\left\{p+\frac{1}{b}\left(1+\frac{z\left(\mathscr{F}_{p, \eta, m, k}(z)\right)^{\prime \prime}}{\left(\mathscr{F}_{p, \eta, m, k}(z)\right)^{\prime}}-p\right)\right\} \\
=\sum_{j=1}^{\eta} k_{j} \mathfrak{R}\left\{p+\frac{1}{b}\left(\frac{z\left(D_{\lambda, l, p}^{m_{j}, \alpha} f_{j}(t)\right)^{\prime}}{D_{\lambda, l, p}^{m_{j}, \alpha} f_{j}(t)}-p\right)\right\} \\
+p-p \sum_{j=1}^{\eta} k_{j} \\
>\sum_{j=1}^{\eta} k_{j} \delta_{j}\left|\frac{1}{b}\left(\frac{z\left(D_{\lambda, l, p}^{m_{j}, \alpha} f_{j}(t)\right)^{\prime}}{D_{\lambda, l, p}^{m_{j}, \alpha} f_{j}(t)}-p\right)\right| \\
+p+\sum_{j=1}^{\eta} k_{j}\left(\beta_{j}-p\right) .
\end{aligned}
$$

Since

$$
\sum_{j=1}^{\eta} k_{j} \delta_{j}\left|\frac{1}{b}\left(\frac{z\left(D_{\lambda, l, p}^{m_{j}, \alpha} f_{j}(t)\right)^{\prime}}{D_{\lambda, l, p}^{m_{j}, \alpha} f_{j}(t)}-p\right)\right|>0
$$

the integral operator $\mathscr{F}_{p, \eta, m, k} \in \mathscr{K}^{p, n}(\tau, b)$ with

$$
\tau=p+\sum_{j=1}^{\eta} k_{j}\left(\beta_{j}-p\right) .
$$

Corollary 8. In Theorem 7, letting

(i) $p=n=1, \delta_{j}=0$, and $m_{j}=0(1 \leq j \leq \eta)$, one obtains [10, Theorem 1].

(ii) $p=n=1, \delta_{j}=0$, and $m_{j}=0, \beta_{j}=\beta \in[0,1)(1 \leq$ $j \leq \eta$ ), one obtains [11, Theorem 1].

(iii) $p=n=1, \delta_{j}=0$, and $m_{j}=0, \beta_{j}=0(1 \leq j \leq \eta)$, one obtains [12, Theorem 1].

Putting $\eta=1, m_{1}=m, k_{1}=k, \beta_{1}=\beta, \delta_{1}=\delta$, and $f_{1}=f$ in Theorem 7, we have the following.

Corollary 9. Let $m \in \mathbb{N}_{0}$ and $k>0$. Also let $b \in \mathbb{C}-\{0\}$, $\delta \geq 0,0 \leq \beta<p$, and $f \in \mathcal{U} \mathcal{S}_{\alpha, \lambda, l}^{m, p, n}(\delta, \beta, b)$. If

$$
0 \leq p+k(\beta-p)<p
$$

then the integral operator $\mathscr{F}_{p, m, k}$, defined by (32), is in the class $\mathscr{K}^{p, n}(\mu, b)$, where

$$
\mu=p+k(\beta-p)
$$

Putting $p=n=1$ and $m=0$ in Corollary 9, we have the following.
Corollary 10. Let $k>0, b \in \mathbb{C}-\{0\}, \delta \geq 0,0 \leq \beta<1$, and $f \in \mathcal{S}(\delta, \beta, b)$. If

$$
0 \leq 1+k(\beta-1)<1,
$$

then the integral operator

$$
\mathscr{F}_{k}(z)=\int_{0}^{z}\left(\frac{f(t)}{t}\right)^{k} d t
$$

is convex of complex order $b(b \in \mathbb{C}-\{0\})$ and type $\rho=1+$ $k(\beta-1)$; that is, $\mathscr{F}_{k} \in \mathscr{K}(\rho, b)$.

Putting $p=n=1, m_{j}=0(1 \leq j \leq \eta)$, and $b=1$ in Theorem 7 , we have the following.

Corollary 11. Let $\eta \in \mathbb{N}, k=\left(k_{1}, \ldots, k_{\eta}\right) \in \mathbb{R}_{+}^{\eta}, \delta_{j} \geq 0$, $0 \leq \beta_{j}<1$, and $f_{j} \in \mathcal{S} \mathscr{D}\left(\delta_{j}, \beta_{j}\right)$ for $1 \leq j \leq \eta$. If

$$
0 \leq 1+\sum_{j=1}^{\eta} k_{j}\left(\beta_{j}-1\right)<1,
$$

then the integral operator

$$
\mathscr{F}(z)=\int_{0}^{z}\left(\frac{f_{1}(t)}{t}\right)^{k_{1}} \cdots\left(\frac{f_{n}(t)}{t}\right)^{k_{n}} d t
$$

is in the class $\mathscr{K}(\sigma)$, where

$$
\sigma=1+\sum_{j=1}^{\eta} k_{j}\left(\beta_{j}-1\right) .
$$

Theorem 12. Let $\eta \in \mathbb{N}, m=\left(m_{1}, \ldots, m_{\eta}\right) \in \mathbb{N}_{0}^{\eta}$, and $k=$ $\left(k_{1}, \ldots, k_{\eta}\right) \in \mathbb{R}_{+}^{\eta}$. Also let $|\theta|<\pi / 2,0 \leq \beta_{j}<p$, and $f_{j} \in$ $\mathcal{S}_{\alpha, \lambda, l}^{m_{j}, p, n}\left(\beta_{j}, \theta\right)$ for $1 \leq j \leq \eta$. If

$$
0 \leq p+\sum_{j=1}^{\eta} k_{j}\left(\beta_{j}-p\right)<p,
$$

then the integral operator $\mathscr{F}_{p, \eta, m, k}$, defined by (29), is in the class $\mathscr{K}^{p, n}(\tau, \theta)$, where

$$
\tau=p+\sum_{j=1}^{\eta} k_{j}\left(\beta_{j}-p\right) .
$$

Proof. From (24), (25), and (40), we get the desired result.

Putting $\eta=1, m_{1}=m, k_{1}=k, \beta_{1}=\beta$ and $f_{1}=f$ in Theorem 12, we have the following.

Corollary 13. Let $m \in \mathbb{N}_{0}$ and $k>0$. Also let $|\theta|<\pi / 2$, $0 \leq \beta<p$, and $f \in \mathcal{S}_{\alpha, \lambda, l}^{m, p, n}(\beta, \theta)$. If

$$
0 \leq p+k(\beta-p)<p
$$

then the integral operator $\mathscr{F}_{p, m, k}$, defined by (32), is in the class $\mathscr{K}^{p, n}(\mu, \theta)$, where

$$
\mu=p+k(\beta-p)
$$


Putting $p=n=1$ and $m=0$ in Corollary 13, we have the following.

Corollary 14. Let $k>0,|\theta|<\pi / 2,0 \leq \beta<1$, and $f \in \mathcal{S}_{\beta}^{\theta}$. If

$$
0 \leq 1+k(\beta-1)<1 \text {, }
$$

then the integral operator $\mathscr{F}_{k}$, defined by (49), is in the class $\mathscr{K}_{\rho}^{\theta}$, where

$$
\rho=1+k(\beta-1) .
$$

Putting $p=n=1$ and $m_{j}=0 \quad(1 \leq j \leq \eta)$ in Theorem 12, we have the following.

Corollary 15. Let $\eta \in \mathbb{N}, k=\left(k_{1}, \ldots, k_{\eta}\right) \in \mathbb{R}_{+}^{\eta}, 0 \leq \beta_{j}<1$, and $f_{j} \in \mathcal{S}_{\beta_{j}}^{\theta}(|\theta|<\pi / 2)$ for $1 \leq j \leq \eta$. If

$$
0 \leq 1+\sum_{j=1}^{\eta} k_{j}\left(\beta_{j}-1\right)<1,
$$

then the integral operator $\mathscr{F}$, defined by (51), is in the class $\mathscr{K}_{\sigma}^{\theta}$, where

$$
\sigma=1+\sum_{j=1}^{\eta} k_{j}\left(\beta_{j}-1\right) .
$$

Theorem 16. Let $\eta \in \mathbb{N}, m=\left(m_{1}, \ldots, m_{\eta}\right) \in \mathbb{N}_{0}^{\eta}$, and $k=$ $\left(k_{1}, \ldots, k_{\eta}\right) \in \mathbb{R}_{+}^{\eta}$. Also let $b \in \mathbb{C}-\{0\}, \delta_{j} \geq 0$, and $0 \leq \beta_{j}<p$ for $1 \leq j \leq \eta$. If

$$
\left|\frac{z\left(D_{\lambda, l, p}^{m_{j}, \alpha} f_{j}(t)\right)^{\prime}}{D_{\lambda, l, p}^{m_{j}, \alpha} f_{j}(t)}-p\right|>-\frac{p+\sum_{j=1}^{\eta} k_{j}\left(\beta_{j}-p\right)}{(1 /|b|) \sum_{j=1}^{\eta} k_{j} \delta_{j}}
$$

for all $1 \leq j \leq \eta$, then the integral operator $\mathscr{F}_{p, \eta, m, k}$, defined by (29), is p-valently convex of complex order $b(b \in \mathbb{C}-\{0\})$; that is, $\mathscr{F}_{p, \eta, m, k} \in \mathscr{K}^{p, n}(b)$.

Proof. From (43) and (61), we easily get that the integral operator $\mathscr{F}_{p, \eta, m, k}$ is $p$-valently convex of complex order $b$.

\section{Sufficient Conditions for \\ the Operator $\mathscr{G}_{p, \eta, m, k}$}

Theorem 17. Let $\eta \in \mathbb{N}, m=\left(m_{1}, \ldots, m_{\eta}\right) \in \mathbb{N}_{0}^{\eta}$, and $k=$ $\left(k_{1}, \ldots, k_{\eta}\right) \in \mathbb{R}_{+}^{\eta}$. Also let $b \in \mathbb{C}-\{0\}, \delta_{j} \geq 0,0 \leq \beta_{j}<p$, and $f_{j} \in \mathcal{U} \mathscr{K}_{\alpha, \lambda, l}^{m_{j}, p, n}\left(\delta_{j}, \beta_{j}, b\right)$ for $1 \leq j \leq \eta$. If

$$
0 \leq p+\sum_{j=1}^{\eta} k_{j}\left(\beta_{j}-p\right)<p,
$$

then the integral operator $\mathscr{G}_{p, \eta, m, k}$, defined by (31), is in the class $\mathscr{K}^{p, n}(\tau, b)$, where

$$
\tau=p+\sum_{j=1}^{\eta} k_{j}\left(\beta_{j}-p\right) .
$$

Proof. From the definition (31), we observe that $\mathscr{G}_{p, \eta, m, k}(z) \in$ $\mathscr{A}_{p}(n)$. On the other hand, it is easy to see that

$$
\left(\mathscr{G}_{p, \eta, m, k}(z)\right)^{\prime}=p z^{p-1} \prod_{j=1}^{\eta}\left(\frac{\left(D_{\lambda, l, p}^{m_{j}, \alpha} f_{j}(z)\right)^{\prime}}{p z^{p-1}}\right)^{k_{j}} .
$$

Differentiating (64) logarithmically and multiplying by $z$, we obtain

$$
\begin{aligned}
& \frac{z\left(\mathscr{G}_{p, \eta, m, k}(z)\right)^{\prime \prime}}{\left(\mathscr{G}_{p, \eta, m, k}(z)\right)^{\prime}} \\
& \quad=(p-1)+\sum_{j=1}^{\eta} k_{j}\left(\frac{z\left(D_{\lambda, l, p}^{m_{j}, \alpha} f_{j}(t)\right)^{\prime \prime}}{\left(D_{\lambda, l, p}^{m_{j}, \alpha} f_{j}(t)\right)^{\prime}}-(p-1)\right)
\end{aligned}
$$

or equivalently

$$
\begin{aligned}
1+ & \frac{z\left(\mathscr{G}_{p, \eta, m, k}(z)\right)^{\prime \prime}}{\left(\mathscr{G}_{p, \eta, m, k}(z)\right)^{\prime}}-p \\
& =\sum_{j=1}^{\eta} k_{j}\left(\frac{z\left(D_{\lambda, l, p}^{m_{j}, \alpha} f_{j}(t)\right)^{\prime \prime}}{\left(D_{\lambda, l, p}^{m_{j}, \alpha} f_{j}(t)\right)^{\prime}}-(p-1)\right) .
\end{aligned}
$$

Then, by multiplying (66) with $1 / b$, we have

$$
\begin{aligned}
\frac{1}{b}(1 & \left.+\frac{z\left(\mathscr{G}_{p, \eta, m, k}(z)\right)^{\prime \prime}}{\left(\mathscr{G}_{p, \eta, m, k}(z)\right)^{\prime}}-p\right) \\
& =\sum_{j=1}^{\eta} k_{j} \frac{1}{b}\left(\frac{z\left(D_{\lambda, l, p}^{m_{j}, \alpha} f_{j}(t)\right)^{\prime \prime}}{\left(D_{\lambda, l, p}^{m_{j}, \alpha} f_{j}(t)\right)^{\prime}}-(p-1)\right)
\end{aligned}
$$

or equivalently

$$
\begin{aligned}
p+ & \frac{1}{b}\left(1+\frac{z\left(\mathscr{G}_{p, \eta, m, k}(z)\right)^{\prime \prime}}{\left(\mathscr{G}_{p, \eta, m, k}(z)\right)^{\prime}}-p\right) \\
= & \sum_{j=1}^{\eta} k_{j}\left(p+\frac{1}{b}\left(1+\frac{z\left(D_{\lambda, l, p}^{m_{j}, \alpha} f_{j}(t)\right)^{\prime \prime}}{\left(D_{\lambda, l, p}^{m_{j}, \alpha} f_{j}(t)\right)^{\prime}}-p\right)\right) \\
& +p-p \sum_{j=1}^{\eta} k_{j} .
\end{aligned}
$$


Since $f_{j} \in \mathscr{U}_{\alpha, \lambda, l}^{m_{j}, p, n}\left(\delta_{j}, \beta_{j}, b\right)(1 \leq j \leq \eta)$, we get

$$
\begin{aligned}
\Re & \left\{p+\frac{1}{b}\left(1+\frac{z\left(\mathscr{G}_{p, \eta, m, k}(z)\right)^{\prime \prime}}{\left(\mathscr{G}_{p, \eta, m, k}(z)\right)^{\prime}}-p\right)\right\} \\
= & \sum_{j=1}^{\eta} k_{j} \mathfrak{R}\left\{p+\frac{1}{b}\left(1+\frac{z\left(D_{\lambda, l, p}^{m_{j}, \alpha} f_{j}(t)\right)^{\prime \prime}}{\left(D_{\lambda, l, p}^{m_{j}, \alpha} f_{j}(t)\right)^{\prime}}-p\right)\right\} \\
& +p-p \sum_{j=1}^{\eta} k_{j} \\
> & \sum_{j=1}^{\eta} k_{j} \delta_{j}\left|\frac{1}{b}\left(1+\frac{z\left(D_{\lambda, l, p}^{m_{j}, \alpha} f_{j}(t)\right)^{\prime \prime}}{\left(D_{\lambda, l, p}^{m_{j}, \alpha} f_{j}(t)\right)^{\prime}}-p\right)\right| \\
& +p+\sum_{j=1}^{\eta} k_{j}\left(\beta_{j}-p\right) .
\end{aligned}
$$

Since

$$
\sum_{j=1}^{\eta} k_{j} \delta_{j}\left|\frac{1}{b}\left(1+\frac{z\left(D_{\lambda, l, p}^{m_{j}, \alpha} f_{j}(t)\right)^{\prime \prime}}{\left(D_{\lambda, l, p}^{m_{j}, \alpha} f_{j}(t)\right)^{\prime}}-p\right)\right|>0,
$$

the integral operator $\mathscr{G}_{p, \eta, m, k} \in \mathscr{K}^{p, n}(\tau, b)$ with

$$
\tau=p+\sum_{j=1}^{\eta} k_{j}\left(\beta_{j}-p\right) .
$$

Corollary 18. In Theorem 17 , letting

(i) $p=n=1, \delta_{j}=0$, and $m_{j}=0(1 \leq j \leq \eta)$, one obtains [10, Theorem 3].

(ii) $p=n=1, \delta_{j}=0$, and $m_{j}=0, \beta_{j}=\beta \in[0,1)(1 \leq$ $j \leq \eta)$, one obtains [11, Theorem 3].

(iii) $p=n=1, \delta_{j}=0$, and $m_{j}=0, \beta_{j}=0(1 \leq j \leq \eta)$, one obtains [12, Theorem 2].

Putting $\eta=1, m_{1}=m, k_{1}=k, \beta_{1}=\beta, \delta_{1}=\delta$, and $f_{1}=f$ in Theorem 17, we have the following.

Corollary 19. Let $m \in \mathbb{N}_{0}$ and $k>0$. Also let $b \in \mathbb{C}-\{0\}$, $\delta \geq 0,0 \leq \beta<p$ and $f \in \mathscr{U}_{\alpha, \lambda, l}^{m, p, n}(\delta, \beta, b)$. If

$$
0 \leq p+k(\beta-p)<p
$$

then the integral operator $\mathscr{G}_{p, m, k}$, defined by (33), is in the class $\mathscr{K}^{p, n}(\mu, b)$, where

$$
\mu=p+k(\beta-p)
$$

Putting $p=n=1$ and $m=0$ in Corollary 19, we have the following.
Corollary 20. Let $k>0, b \in \mathbb{C}-\{0\}, \delta \geq 0,0 \leq \beta<1$, and $f \in \mathscr{K}(\delta, \beta, b)$. If

$$
0 \leq 1+k(\beta-1)<1
$$

then the integral operator

$$
\mathscr{G}_{k}(z)=\int_{0}^{z}\left(f^{\prime}(t)\right)^{k} d t
$$

is convex of complex order $b(b \in \mathbb{C}-\{0\})$ and type $\rho=1+$ $k(\beta-1)$; that is, $\mathscr{G}_{k} \in \mathscr{K}(\rho, b)$.

Putting $p=n=1, m_{j}=0(1 \leq j \leq \eta)$, and $b=1$ in Theorem 17, we have the following.

Corollary 21. Let $\eta \in \mathbb{N}, k=\left(k_{1}, \ldots, k_{\eta}\right) \in \mathbb{R}_{+}^{\eta}, \delta_{j} \geq 0$, $0 \leq \beta_{j}<1$, and $f_{j} \in \mathscr{K} \mathscr{D}\left(\delta_{j}, \beta_{j}\right)$ for $1 \leq j \leq \eta$. If

$$
0 \leq 1+\sum_{j=1}^{\eta} k_{j}\left(\beta_{j}-1\right)<1,
$$

then the integral operator

$$
\mathscr{G}(z)=\int_{0}^{z}\left(f_{1}^{\prime}(t)\right)^{k_{1}} \cdots\left(f_{n}^{\prime}(t)\right)^{k_{n}} d t
$$

is in the class $\mathscr{K}(\sigma)$, where

$$
\sigma=1+\sum_{j=1}^{\eta} k_{j}\left(\beta_{j}-1\right) .
$$

Theorem 22. Let $\eta \in \mathbb{N}, m=\left(m_{1}, \ldots, m_{\eta}\right) \in \mathbb{N}_{0}^{\eta}$, and $k=$ $\left(k_{1}, \ldots, k_{\eta}\right) \in \mathbb{R}_{+}^{\eta}$. Also let $|\theta|<\pi / 2,0 \leq \beta_{j}<p$, and $f_{j} \in$ $\mathscr{K}_{\alpha, \lambda, l}^{m_{j}, p, n}\left(\beta_{j}, \theta\right)$ for $1 \leq j \leq \eta$. If

$$
0 \leq p+\sum_{j=1}^{\eta} k_{j}\left(\beta_{j}-p\right)<p,
$$

then the integral operator $\mathscr{G}_{p, \eta, m, k}$, defined by (31), is in the class $\mathscr{K}^{p, n}(\tau, \theta)$, where

$$
\tau=p+\sum_{j=1}^{\eta} k_{j}\left(\beta_{j}-p\right)
$$

Proof. From (25) and (66), we get the desired result.

Putting $\eta=1, m_{1}=m, k_{1}=k, \beta_{1}=\beta$, and $f_{1}=f$ in Theorem 22, we have the following.

Corollary 23. Let $m \in \mathbb{N}_{0}$ and $k>0$. Also let $|\theta|<\pi / 2$, $0 \leq \beta<p$, and $f \in \mathscr{K}_{\alpha, \lambda, l}^{m, p, n}(\beta, \theta)$. If

$$
0 \leq p+k(\beta-p)<p
$$

then the integral operator $\mathscr{G}_{p, m, k}$, defined by (33), is in the class $\mathscr{K}^{p, n}(\mu, \theta)$, where

$$
\mu=p+k(\beta-p)
$$


Putting $p=n=1$ and $m=0$ in Corollary 23, we have the following.

Corollary 24. Let $k>0,|\theta|<\pi / 2,0 \leq \beta<1$, and $f \in \mathscr{K}_{\beta}^{\theta}$. If

$$
0 \leq 1+k(\beta-1)<1
$$

then the integral operator $\mathscr{G}_{k}$, defined by (75), is in the class $\mathscr{K}_{\rho}^{\theta}$, where

$$
\rho=1+k(\beta-1) .
$$

Putting $p=n=1$ and $m_{j}=0(1 \leq j \leq \eta)$ in Theorem 22, we have the following.

Corollary 25. Let $\eta \in \mathbb{N}, k=\left(k_{1}, \ldots, k_{\eta}\right) \in \mathbb{R}_{+}^{\eta}, 0 \leq \beta_{j}<1$, and $f_{j} \in \mathscr{K}_{\beta_{j}}^{\theta}(|\theta|<\pi / 2)$ for $1 \leq j \leq \eta$. If

$$
0 \leq 1+\sum_{j=1}^{\eta} k_{j}\left(\beta_{j}-1\right)<1
$$

then the integral operator $\mathscr{G}$, defined by (77), is in the class $\mathscr{K}_{\sigma}^{\theta}$, where

$$
\sigma=1+\sum_{j=1}^{\eta} k_{j}\left(\beta_{j}-1\right)
$$

Theorem 26. Let $\eta \in \mathbb{N}, m=\left(m_{1}, \ldots, m_{\eta}\right) \in \mathbb{N}_{0}^{\eta}$, and $k=$ $\left(k_{1}, \ldots, k_{\eta}\right) \in \mathbb{R}_{+}^{\eta}$. Also let $b \in \mathbb{C}-\{0\}, \delta_{j} \geq 0$, and $0 \leq \beta_{j}<p$ for $1 \leq j \leq \eta$. If

$$
\left|1+\frac{z\left(D_{\lambda, l, p}^{m_{j}, \alpha} f_{j}(t)\right)^{\prime \prime}}{\left(D_{\lambda, l, p}^{m_{j}, \alpha} f_{j}(t)\right)^{\prime}}-p\right|>-\frac{p+\sum_{j=1}^{\eta} k_{j}\left(\beta_{j}-p\right)}{(1 /|b|) \sum_{j=1}^{\eta} k_{j} \delta_{j}}
$$

for all $1 \leq j \leq \eta$, then the integral operator $\mathscr{G}_{p, \eta, m, k}$, defined by (31), is p-valently convex of complex order $b(b \in \mathbb{C}-\{0\})$; that is, $\mathscr{G}_{p, \eta, m, k} \in \mathscr{K}^{p, n}(b)$.

Proof. From (69) and (87), we easily get that the integral operator $\mathscr{G}_{p, \eta, m, k}$ is $p$-valently convex of complex order $b$.

\section{References}

[1] S. Bulut, "The generalization of the generalized Al-Oboudi differential operator," Applied Mathematics and Computation, vol. 215, no. 4, pp. 1448-1455, 2009.

[2] S. Bulut, "Coefficient inequalities for certain subclasses of analytic functions defined by using a general derivative operator," Kyungpook Mathematical Journal, vol. 51, no. 3, pp. 241-250, 2011.

[3] S. Shams, S. R. Kulkarni, and J. M. Jahangiri, "Classes of uniformly starlike and convex functions," International Journal of Mathematics and Mathematical Sciences, vol. 55, no. 53-56, pp. 2959-2961, 2004.

[4] F. Rønning, "On starlike functions associated with parabolic regions," Annales Universitatis Mariae Curie-Skłodowska. A. Mathematica, vol. 45, pp. 117-122, 1991.
[5] A. W. Goodman, "On uniformly convex functions," Annales Polonici Mathematici, vol. 56, no. 1, pp. 87-92, 1991.

[6] R. J. Libera, "Univalent $\alpha$-spiral functions," Canadian Journal of Mathematics, vol. 19, pp. 449-456, 1967.

[7] P. N. Chichra, "Regular functions $f(z)$ for which $z f^{\prime}(z)$ is $\alpha$ spiral-like," Proceedings of the American Mathematical Society, vol. 49, pp. 151-160, 1975.

[8] G. Saltık, E. Deniz, and E. Kadığlu, “Two new general p-valent integral operators," Mathematical and Computer Modelling, vol. 52, no. 9-10, pp. 1605-1609, 2010.

[9] B. A. Frasin, "New general integral operators of $p$-valent functions," Journal of Inequalities in Pure and Applied Mathematics, vol. 10, no. 4, article 109, p. 9, 2009.

[10] S. Bulut, "A note on the paper of Breaz and Güney," Journal of Mathematical Inequalities, vol. 2, no. 4, pp. 549-553, 2008.

[11] D. Breaz and H. Ö. Güney, "The integral operator on the classes $\mathcal{S}_{\alpha}^{*}(b)$ and $C_{\alpha}(b)$," Journal of Mathematical Inequalities, vol. 2, no. 1, pp. 97-100, 2008.

[12] D. Breaz, M. K. Aouf, and N. Breaz, "Some properties for integral operators on some analytic functions with complex order," Acta Mathematica. Academiae Paedagogicae Nyíregyháziensis. New Series, vol. 25, no. 1, pp. 39-43, 2009. 


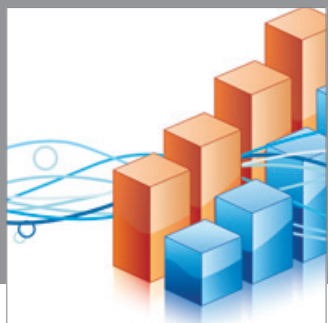

Advances in

Operations Research

mansans

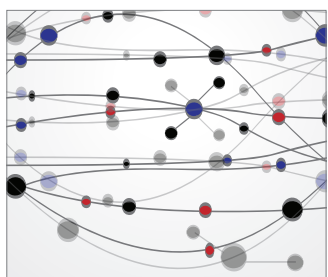

The Scientific World Journal
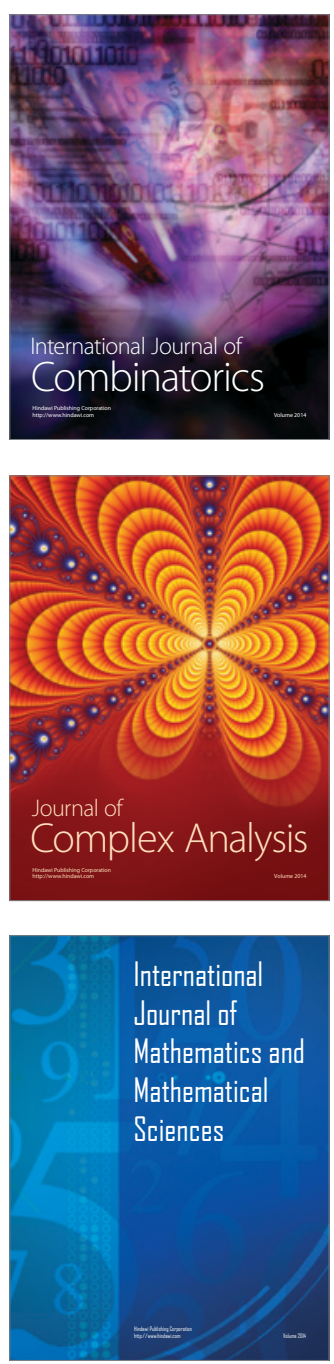
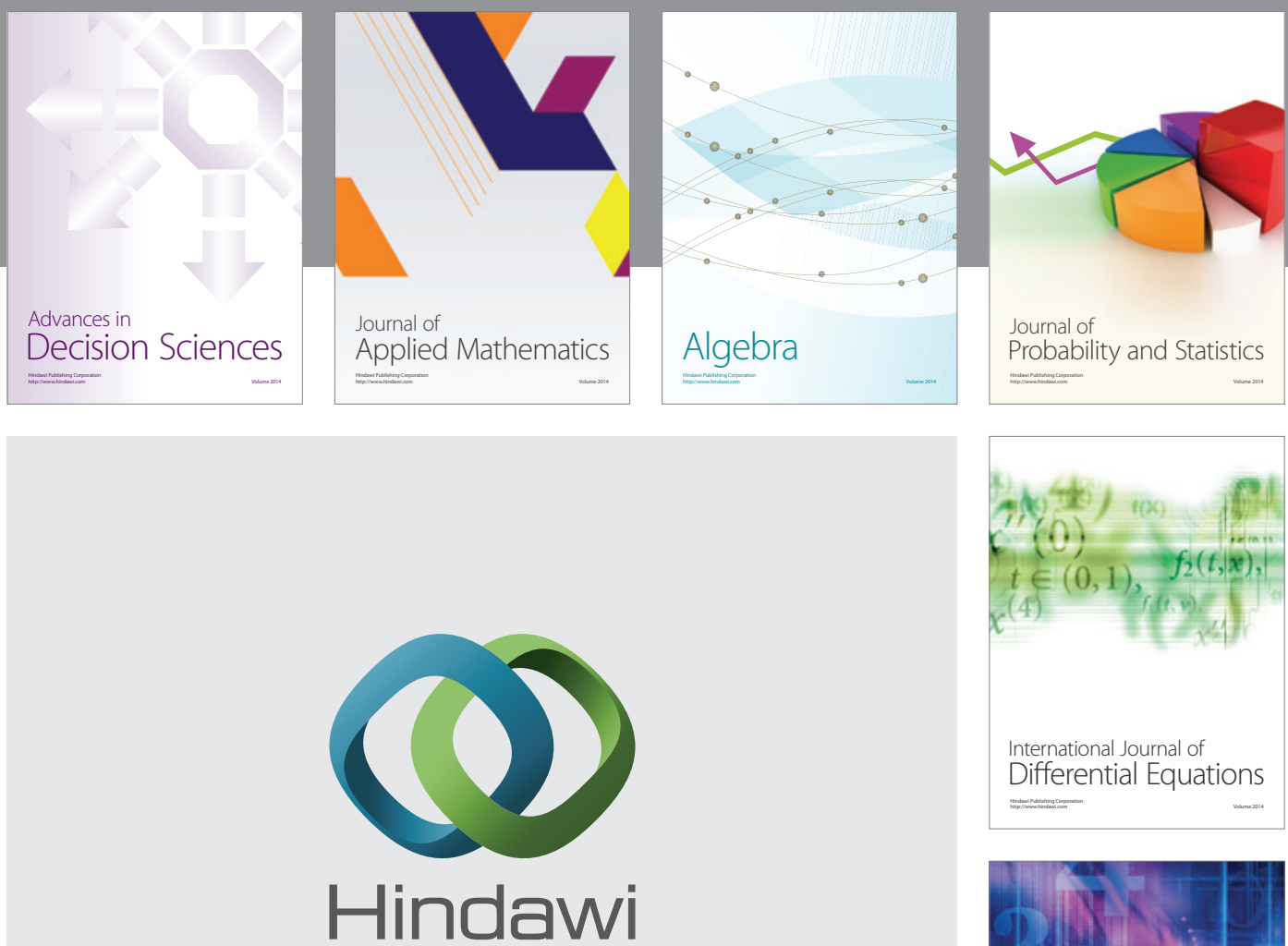

Submit your manuscripts at http://www.hindawi.com
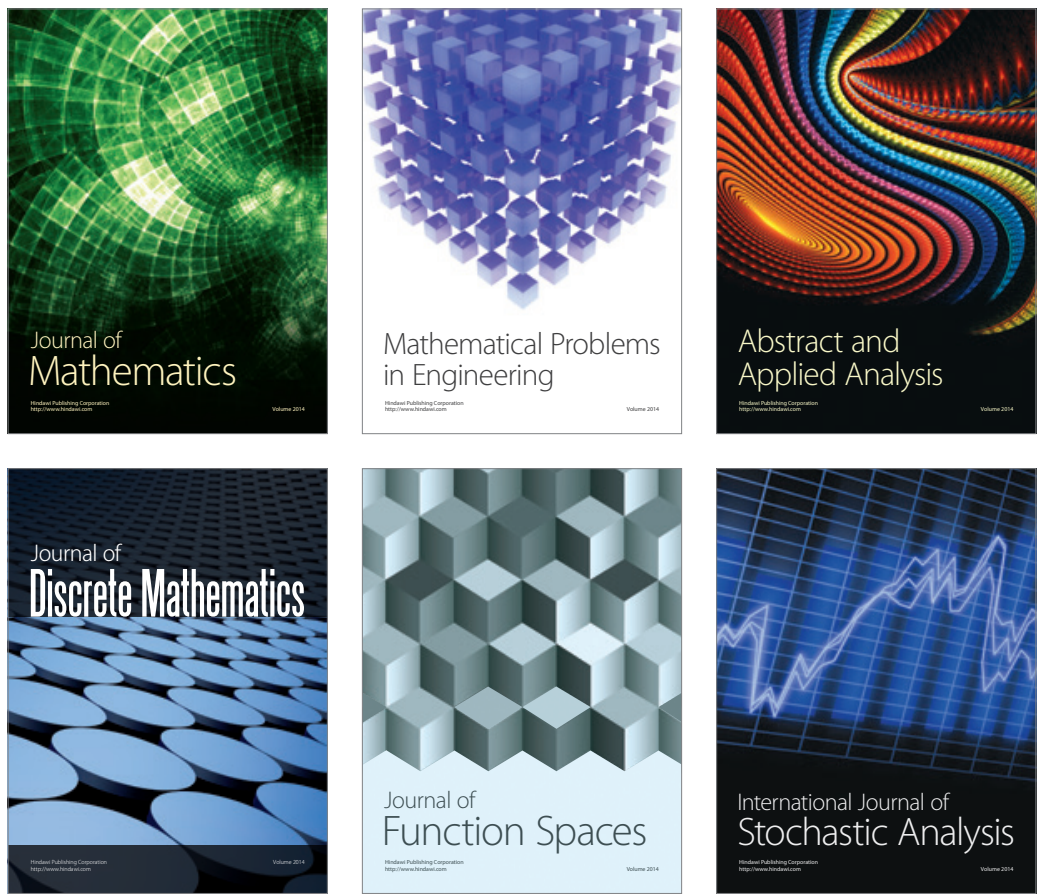

Journal of

Function Spaces

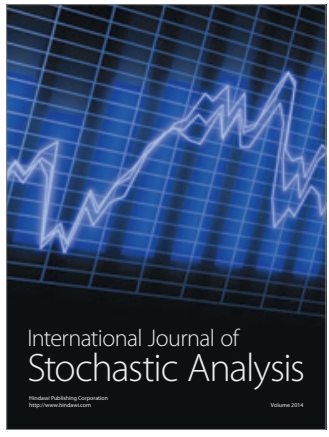

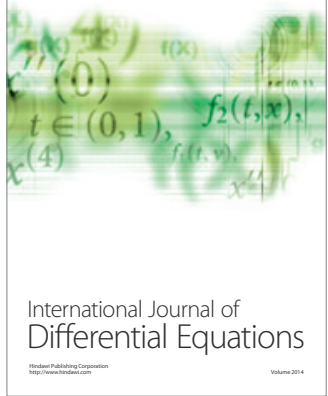
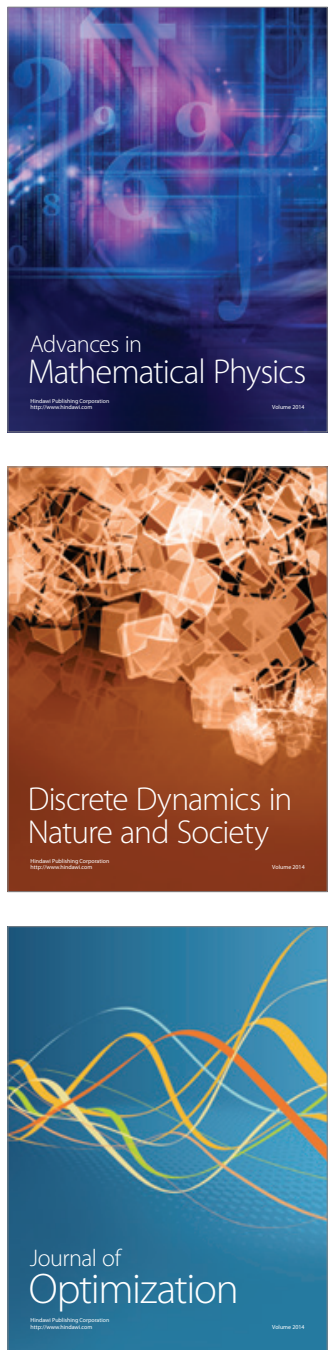